\title{
Caracterização molecular de acessos de Cratylia argentea e sua relação filogenética com outras leguminosas
}

\author{
Alexsandro Sobreira Galdino(1), João Paulo Matos Santos Lima(2), Renata de Souza Panarari Antunes(3), \\ José Alberto Prioli( ${ }^{(4)}$, Paulo Roberto Thiers ${ }^{(5)}$, Glocimar Pereira da Silva ${ }^{(6)}$ e Thalles Barbosa Grangeiro(5)
}

\begin{abstract}
(1)Universidade Federal de São João Del-Rei, Avenida Sebastião Gonçalves Coelho oo 400, Chanadour, CEP $35501-296$ Divinópolis, MG. E-mail:asgaldino@ufsj.edu.br(2)UniversidadeFederaldoRio GrandedoNorte,AvenidaSalgadoFilho,s/no, LagoaNova, CEP59078-970Natal, RN. E-mail: jpmatos@cb.ufrn.br ${ }^{(3)}$ Instituto Federal do Paraná, Trevo Codapar, s/nº, CEP 85555-000 Palmas, PR. E-mail: panarari@gmail.com (4)Universidade Estadual de Maringá, Avenida Colombo no 5790, CEP 87020-900 Maringá, PR. E-mail: ajprioli@nupelia.uem.br (5)Universidade

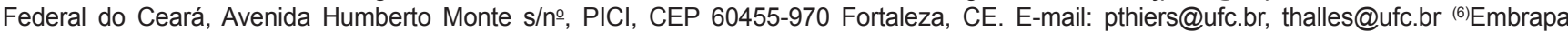
Recursos Genéticos e Biotecnologia, Parque Estação Biológica, Asa Norte, CEP 70770-900 Brasília, DF. E-mail: glocimar@cenargen.embrapa.br
\end{abstract}

Resumo - O objetivo deste trabalho foi realizar a caracterização molecular de 11 acessos de Cratylia argentea, com base no sequenciamento da região ITS (ITS1/5,8S/ITS2), bem como o estabelecimento de suas relações filogenéticas com outras leguminosas. As relações filogenéticas dessa espécie com outras 15 leguminosas foram estabelecidas com o uso de sequência do gene que codifica a subunidade 18S do rRNA (rDNA 18S). A amplificação do DNA da região ITS $/ 5,8 \mathrm{~S}$ dos 11 acessos revelou uma única banda de aproximadamente $650 \mathrm{pb}$. Sequências ITS $/ 5,8 \mathrm{~S}$ foram obtidas de todos os acessos analisados e depois alinhadas com a região ITS $/ 5,8 \mathrm{~S}$ da leguminosa Galactia striata. O tamanho das sequências ITS $/ 5,8 \mathrm{~S}$ dos acessos de $C$. argentea variou de 565 a 615 pb. Os conteúdos médios de G + C nas regiões ITS1 e ITS2 variaram entre 46 e 47\%. $\mathrm{O}$ alinhamento múltiplo das seqüências ITS $/ 5,8 \mathrm{~S}$ dos acessos de C. argentea com Galactia striata revelou a presença de deleções e inserções. Os acessos de $C$. argentea constituíram um único clado politômico. A análise filogenética de $C$. argentea demonstrou que essa espécie está incluída no clado das Diocleinae verdadeiras e que os gêneros Calopogonium e Pachyrhizus estão fora desse clado.

Termos para indexação: distância genética, forrageiras, leguminosas arbustivas, variabilidade genética.

\section{Molecular characterization of Cratylia argentea accessions and its phylogenetic relationship with other legumes}

\begin{abstract}
The objective of this work was to molecularly characterize 11 Cratylia argentea accessions, based on the ITS (ITS1/5.8S/ITS2) region sequencing, as well to establish its phylogenetic relationship with other legume species. The phylogenetic relationship of this species with other 15 legume ones was established using a gene sequence that codes the subunit $18 \mathrm{~S}$ of the rRNA (rDNA 18S). DNA amplification of the ITS/5.8S region of these 11 accessions revealed an amplicon with around $650 \mathrm{bp}$. ITS/5.8S sequences were obtained from all accessions analysed, and then aligned with the region ITS/5.8S of Galactia striata legume. The size of ITS/5.8S region ranged from 565 to $615 \mathrm{bp}$. Average $\mathrm{G}+\mathrm{C}$ contents in the ITS1 and ITS2 regions ranged between 46 and $47 \%$. The multiple sequence alignment between the ITS sequences from $C$. argentea accessions and Galactia striata revealed the presence of deletions and insertions. C. argentea accessions formed a unique politomic clade. Cratylia argentea phylogenetic analysis demonstrated that this species is placed into the true Diocleinae Clade, and that Calopogonium and Pachyrhizus are not included in subtribe Diocleinae.
\end{abstract}

Index terms: genetic distance, forage plant, legume shrubs, genetic variability.

\section{Introdução}

Cratylia (Fabaceae-Papilionoideae-Phaseoleae -Diocleinae) é um gênero neotropical de origem recente, essencialmente extra-amazônico, que pode ser encontrado do Peru até a bacia do rio Paraná (Queiroz \& Coradín, 1995). A faixa altitudinal de ocorrência desse gênero estende-se desde o nível do mar até $950 \mathrm{~m}$. Isso torna possível, em algumas espécies, ultrapassar barreiras fluviais através da cabeceira dos rios (Queiroz \& Coradín, 1995).

A taxonomia do gênero Cratylia ainda está em processo de definição. Trabalhos baseados no estudo de grandes coleções de herbários (Queiroz, 1991) e

Pesq. agropec. bras., Brasília, v.45, n.8, p.846-854, ago. 2010 
trabalhos de campo resultaram no reconhecimento de cinco espécies válidas: Cratylia argentea (Desv.) O. Kuntze; C. bahiensis L.P Queiroz; C. hypargyrea Mart. ex Benth.; C. intermedia (Hassl.) L.P Queiroz; e R. Monteiro e C. mollis Marth ex Benth (Queiroz \& Coradín, 1995). Entre essas, C. argentea destacase como espécie de grande potencial forrageiro, pois possui porte arbustivo, profusamente ramificado, com elevada resistência à seca (Argel \& Lascano, 1998).

Outro fator que merece destaque é a resistência natural dessa espécie a solos ácidos com grande saturação de alumínio (Mass, 1995). Embora C. argentea possua importância econômica e alto valor nutritivo (Argel \& Lascano, 1998), existem poucos estudos sobre sua biologia e genética (Vargas et al., 2007). A caracterização molecular de acessos de C. argentea pode auxiliar o desenvolvimento de novas cultivares, nas quais acessos com maior variabilidade genética podem ser selecionados.

Um instrumento eficiente para a identificação da variabilidade genética entre indivíduos de uma mesma população ou entre indivíduos de populações diferentes é o sequenciamento de regiões gênicas variáveis. Entre essas regiões, destaca-se o sequenciamento das regiões denominadas ITS ("Internal Transcribed Spacers"), presentes no genoma nuclear, e que fazem parte do gene que codifica as subunidades $18 \mathrm{~S}, 5,8 \mathrm{~S}$ e $28 \mathrm{~S}$ do RNA ribossômico. Devido à sua alta taxa de substituição nucleotídica, esses espaçadores têm sido empregados como ferramentas para estudar tanto a diversidade genética como para desenvolver estudos filogenéticos entre plantas (Schmickl et al., 2010) .

A taxonomia do gênero Cratylia está em processo de definição. Queiroz (1991), em análise das grandes coleções de herbários e trabalhos de campo, reconheceu cinco espécies, entre elas $C$. argentea. A diferenciação entre as espécies do gênero Cratylia era baseada, a princípio, apenas em caracteres morfológicos e vegetativos. Atualmente, as análises de dados morfológicos e moleculares têm auxiliado no entendimento de relações filogenéticas entre plantas. Sequências nucleares e extranucleares - DNA genômico de cloroplastos - podem ser utilizadas para tais finalidades, em plantas. A escolha do tipo de sequência depende do objetivo do estudo e do grau de parentesco entre as espécies. As regiões ITS1 e ITS2, por exemplo, podem ser utilizadas entre espécies próximas e também no plano intraespecífico (Mayer
\& Soltis, 1999). No entanto, para estabelecer relações filogenéticas entre espécies distantemente relacionadas, são utilizadas sequências mais conservadas, como as sequências que codificam a subunidade $18 \mathrm{~S}$ ribossomal (rDNA 18S). Por ser uma região bem conservada, o rDNA $18 \mathrm{~S}$ tem sido amplamente empregado em estudos filogenéticos em plantas (Hao et al., 2008; Tokuoka, 2008).

Este trabalho teve como objetivo de realizar a caracterização molecular de 11 acessos de $C$. argentea com base no sequenciamento da região ITS (ITS1/5,8S/ ITS2), bem como o estabelecimento de relações filogenéticas com outras 15 leguminosas, sequenciadas e depositadas no GenBank, utilizando a sequência do rDNA $18 \mathrm{~S}$.

\section{Material e Métodos}

Sementes de 11 acessos de C. argentea, coletados em diferentes municípios do Estado de Goiás e em um município do Estado do Mato Grosso, foram cedidas pelo Banco de Germoplasma da Embrapa Recursos Genéticos e Biotecnologia, Brasília, DF (Tabela 1).

Amostras de DNA genômico de cada acesso foram extraídas de folhas frescas e purificadas, conforme Foster \& Twell (1996). As amplificações in vitro foram realizadas por PCR ("Polimerase Chain Reaction"), segundo Varela et al. (2004). Foram realizadas reações controle para verificar a ocorrência de eventuais amplificações indesejadas. Os primers usados para amplificação da região ITS1/5,8S/ITS2 foram chamados de: ITS 4 ( 5 '-TCCTCCGCTTATTGATATGC-3') e ITS5 (5'-GCAAGTAAAAGTCGTAACAAGG-3') de acordo com Becerra \& Venable (1999). As reações de PCR continham, em um volume final de $25 \mu \mathrm{L}$, DNA genômico (100 ng por reação), tampão Tris-HCl $20 \mathrm{mmol}$ $\mathrm{L}^{-1}$, KCL $50 \mathrm{mmol} \mathrm{L}-1, \mathrm{MgCl}_{2}$ 1,5 $\mathrm{mmol} \mathrm{L}^{-1}$, dNTPs

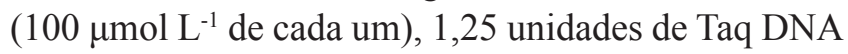
polimerase, e primers $\left(5 \mathrm{mmol} \mathrm{L}^{-1}\right)$. As condições de amplificação consistiram de 30 ciclos de $94^{\circ} \mathrm{C}$, por $1 \mathrm{~min}$, anelamento, a $55^{\circ} \mathrm{C}$, por $1 \mathrm{~min}$, e extensão, a $72^{\circ} \mathrm{C}$, por $2 \mathrm{~min}$.

Para sequenciar a região do rDNA 18S, três grupos de primers foram usados e são, a seguir, relacionados: 18SF1 (5'-GTCATATGCTTGTCTCAAAGA-3'), 18SR1 (5'- CGACCCAACCCAAGGTCC-3'), $18 \mathrm{SF} 2$ (5'-TAATTCCAGCTCCAATAG-3'), 18SR2 (5' -CCACCCATAGAATCAAGA-3') e 
18SF3 (5'-GGCACCACCAGGAGTGGA-3'), 18SR3 (5' -CAATGATCCTTCCGCAGG-3'), conforme descrito por Cunha (2002). Os produtos da PCR foram sequenciados com uso do kit de sequenciamento DYEnamic ET terminators cycle sequencing kit (Amersham Biosciences, Uppsala, Suécia), segundo protocolo descrito pelo fabricante. As sequências obtidas de cada acesso foram alinhadas e a sequência consenso de cada um foi determinada a partir do alinhamento obtido pelo programa ClustalW (Thompson et al., 1994). A confiabilidade dos consensos obtidos foi confirmada por meio de inspeção visual dos eletroferogramas de cada sequência, utilizando o programa BioEdit versão 5.09 (Hall, 1999). Posteriormente, uma sequência consenso para cada sequenciamento foi determinada. Cada sequência consenso foi submetida à busca por similaridade no GenBank (NCBI, National Center for Biotechnology Information), através do programa Blast (Basic Local Aligment Search Tool) (Altschul et al., 1990). A análise de similaridade foi utilizada para escolha do grupo externo. Em seguida, as sequências foram novamente alinhadas pelo Clustal com a sequência do grupo externo selecionado. Após o alinhamento das sequências, foi feita uma análise quanto à saturação de bases, pelo programa Dambe (Data Analyses of Molecular Biology and Evolution) versão 4.065 (Xia \& Xie, 2001).

$\mathrm{Na}$ análise filogenética, foram utilizadas as seguintes sequências do rDNA $18 \mathrm{~S}$ de leguminosas depositadas no GenBank: Bauhinia variegata (AF525295), Calopogonium caeruleum (AF525696), Cleobulia multiflora (AF525697), Cratylia argentea (GenBank AF525294), Cymbosema roseum (AF525694),
Dioclea guianensis (AF525698), Erythrina cristagalli (AF525296), Mucuna pruriens (AF525695), Mucuna sloanei (AF525699), Pachyrhizus erosus (AF525693), Albizzia julibrissin (U42536), Astragalus propinquus (AF359604), Glycine max (X02623), Medicago truncatula (AF093506) e Pisum sativum (U43011). Para essa análise filogenética, foi utilizada a sequência de $C$. argentea (GenBank AF525294) correspondente à sequência $18 \mathrm{~S}$ do acesso 591 . As sequências utilizadas na análise filogenética tinham aproximadamente $1,7 \mathrm{~kb}$. Inicialmente, uma análise prévia com o programa ModelTest (Posada \& Crandall, 1998) foi realizada para verificar qual modelo de substituição se aplicaria às sequência utilizadas.

Em seguida, a análise filogenética, que utilizou as sequências do rDNA $18 \mathrm{~S}$ das leguminosas, foi realizada pelos programas SeqBoot (valor de "bootstrap" de 100), DNADIST (distância de Kimura 2-parâmetros), Neighbor (método Neighbor-joining) e Consense, presentes no pacote Phylip versão 3.5c (Felsenstein, 1993). Na análise filogenética com sequências $18 \mathrm{~S}$, foi selecionado o modelo de substituição nucleotídica Kimura 2-parâmetros (Kimura, 1980). Com a matriz de distâncias genéticas obtidas por meio desse modelo, foi construído o dendrograma "neighbor-joining". A árvore filogenética que relacionou as sequências foi visualizada com o programa TreeView (Page, 1996).

\section{Resultados e Discussão}

Sequências de boa qualidade da região ITS $/ 5,8 \mathrm{~S}$ foram obtidas de todos os acessos analisados de C. argentea. $\mathrm{O}$ tamanho das sequências ITS $/ 5,8 \mathrm{~S}$ variou de 565, no acesso BRA-000604, a 615 pb, no acesso

Tabela 1. Dados de passaporte dos 11 acessos de Cratylia argentea utilizados no presente trabalho.

\begin{tabular}{|c|c|c|c|c|c|c|}
\hline Acesso & Município de coleta $^{(1)}$ & Ambiente específico de coleta & Tipo de solo & Latitude e longitude & Altitude & Sigla \\
\hline BRA-000591 & Posse & Mata seca em afloramento calcário & Argiloso & $14^{\circ} 51^{\prime} \mathrm{S} 46^{\circ} 23^{\prime} \mathrm{W}$ & $780 \mathrm{~m}$ & 591 Pos \\
\hline BRA-000604 & São Domingos & Mata seca em afloramento calcário & Argiloso & $13^{\circ} 30^{\prime} \mathrm{S} 46^{\circ} 24^{\prime} \mathrm{W}$ & $650 \mathrm{~m}$ & $604 \mathrm{SaD}$ \\
\hline BRA-000621 & Campos Belos & Mata seca de beira de estrada & Argiloso & $13^{\circ} 10^{\prime} \mathrm{S} 46^{\circ} 00^{\prime} \mathrm{W}$ & $540 \mathrm{~m}$ & $621 \mathrm{CaB}$ \\
\hline BRA-000639 & Campos Belos & Cerrado mesofítico & Calcário de boa qualidade & $13^{\circ} 15^{\prime} \mathrm{S} 46^{\circ} 28^{\prime} \mathrm{W}$ & $700 \mathrm{~m}$ & $639 \mathrm{CaB}$ \\
\hline BRA-000663 & Mara Rosa & Cerrado antropizado ${ }^{(2)}$ & Areno-argiloso & $13^{\circ} 54^{\prime} \mathrm{S} 49^{\circ} 03^{\prime} \mathrm{W}$ & $510 \mathrm{~m}$ & $663 \mathrm{MaR}$ \\
\hline BRA-000671 & Santa Tereza de Goiás & Cerrado antropizado & Areno-argiloso & $13^{\circ} 48^{\prime} \mathrm{S} 49^{\circ} 23^{\prime} \mathrm{W}$ & $510 \mathrm{~m}$ & $671 \mathrm{STG}$ \\
\hline BRA-000680 & Porangatu & Cerrado antropizado & Calcário de boa qualidade & $13^{\circ} 12^{\prime} \mathrm{S} 49^{\circ} 07^{\prime} \mathrm{W}$ & $380 \mathrm{~m}$ & 680 Por \\
\hline BRA-000698 & Novo Planalto & Cerrado Mata de Galeria & Areno-argiloso & $13^{\circ} 14^{\prime} \mathrm{S} 49^{\circ} 28^{\prime} \mathrm{W}$ & $340 \mathrm{~m}$ & $698 \mathrm{NoP}$ \\
\hline BRA-000701 & São Miguel do Araguaia & Cerrado Mata de Galeria & Areno-argiloso & $13^{\circ} 14^{\prime} \mathrm{S} 50^{\circ} 40^{\prime} \mathrm{W}$ & $360 \mathrm{~m}$ & $701 \mathrm{SMA}$ \\
\hline BRA-000728 & São Miguel do Araguaia & Área degradada & Argilo-arenoso & $13^{\circ} 51^{\prime} \mathrm{S} 50^{\circ} 20^{\prime} \mathrm{W}$ & $300 \mathrm{~m}$ & 728 SMA \\
\hline BRA-000752 & Guiratinga & Cerrado ralo & Arenoso & $16^{\circ} 23^{\prime} \mathrm{S} 54^{\circ} 01^{\prime} \mathrm{W}$ & $330 \mathrm{~m}$ & 752 GUI \\
\hline
\end{tabular}

${ }^{(1)}$ Municípios de coleta em Goiás, exceto Guiratinga, no Mato Grosso. ${ }^{(2)}$ Os ambientes de cerrado antropizado encontram-se próximos a rodovias. 
BRA-000728 (Figura 1). Há relatos sobre regiões ITS $/ 5,8 \mathrm{~S}$ com tamanho menor do que $700 \mathrm{pb}$ em plantas das seguintes famílias das angiospermas: Adoxaceae, Apiaceae, Asteraceae, Betulaceae, Brassicaceae, Canellaceae, Curcubitaceae, Fabaceae, Onagraceae, Malvaceae, Poaceae, Polemiaceae, Ranunculaceae e Rosaceae (Baldwin, 1992; Baldwin et al., 1995).

Yokota et al. (1989), em trabalhos com o sequenciamento de duas espécies de dicotiledôneas,
Vicia faba e Daucus carota, determinaram que o tamanho da região ITS/5,8S foi de $603 \mathrm{pb}$ em ambas as espécies. Os resultados encontrados neste trabalho estão de acordo com os de Yokota et al. (1989), o que é compreensível, uma vez que Vicia faba também pertence à família Fabaceae. De acordo com esses resultados, ambas as espécies estão relacionadas.

Em relação à análise de busca por similaridade em bancos públicos de sequências de DNA, o resultado

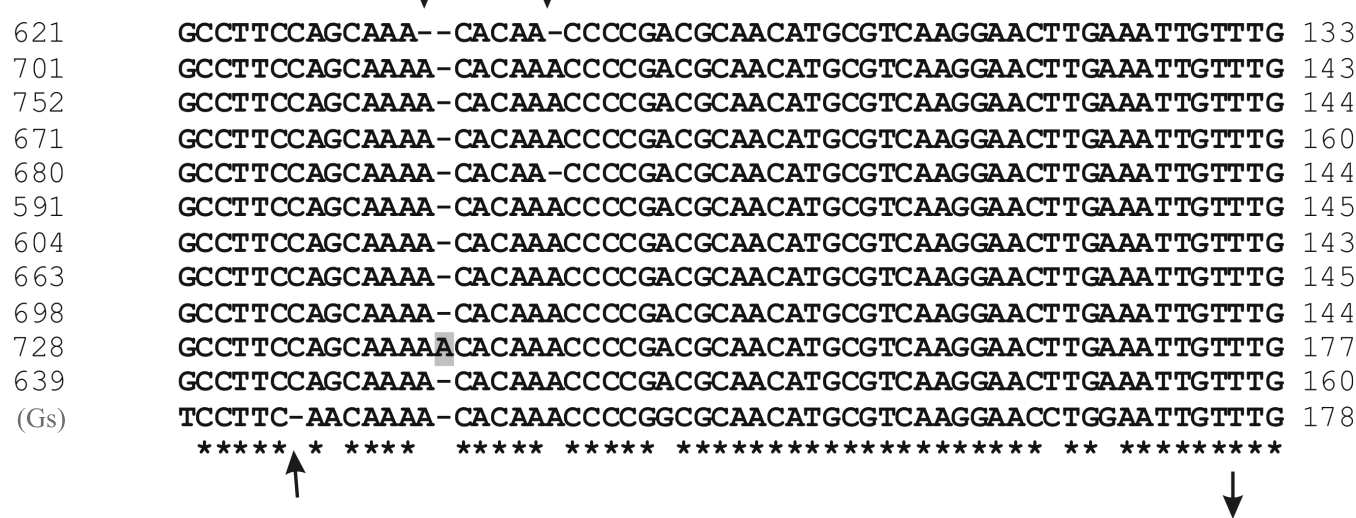

AAATGACTCTCGGCAACGGATATCTCGG-CTCTTGCATCGATGAAGAACGTAGCAAA-TG 251 AAATGACTCTCGGCAACGGATATCTCGG-CTCTTGCATCGATGAAGAACGTAGCAAAATG 262 AAATGACTCTCGGCAACGGATATCTCGG-CTCTTGCATCGATGAAGAACGTAGCAAAATG 263 AAATGACTCTCGGCAACGGATATCTCGG-CTCTTGCATCGATGAAGAACGTAGCAAAATG 279 AAATGACTCTCGGCAACGGATATCTCGG-СTCTTGCATCGATGAAGAACGTAGCAAAATG 263 AAATGACTCTCGGCAACGGATATCTCGG-CTCTTGCATCGATGAAGAACGTAGCAAAATG 264 AAATGACTCTCGGCAACGGATATCTCGG-CTCTTGCATCGATGAAGAACGTAGCAAAATG 262 AAATGACTCTCGGCAACGGATATCTCGGGCTCTTGCATCGATGAAGAACGTAGCAAAATG 265 AAATGACTCTCGGCAACGGATATCTCGG-СTCTTGCATCGATGAAGAACGTAGCAAAATG 263 AAATGACTCTCGGCAACGGATATCTCGG-CTCTTGCATCGATGAAGAACGTAGCAAAATG 296 AAATGACTCTCGGCAACGGATATCTCGG-СTCTTGCATCGATGAAGAACGTAGCAAAATG 279 AAATGACTCTCGGCAACGGATATCTCGG-CTCTTGCATCGATGAAGAACGTAGCAAAATG 296

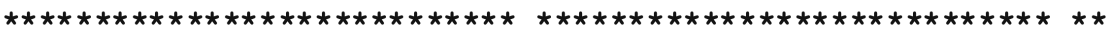

621 CGATACTTGG-TGTGAATTGCA-GAATCCCGTGAACCA-TCGAGTCTTTGAACGCAAGTT 308 CGATACTTGGGTGTGAATTGCA-GAATCCCGTGAACCA-TCGAGTCTTTGAACGCAAGTT 320 CGATACTTGG-TGTGAATTGCA-GAATCCCGTGAACCA-TCGAGTCTTTGAACGCAAGTT 320 CGATACTTGG-TGTGAATTGCA-GAATCCCGTGAACCA-TCGAGTCTTTGAACGCAAGTT 336 CGATACTTGG-TGTGAATTGCA-GAATCCCGTGAACCA-TCGAGTCTTTGAACGCAAGTT 320 CGATACTTGG-TGTGAATTGCA-GAATCCCGTGAACCA-TCGAGTCTTTGAACGCAAGTT 321 CGATACTTGG-TGTGAATTGCA-GAATCCCGTGAACCA-TCGAGTCTTTGAACGCAAGTT 319 CGATACTTGG-TGTGAATTGCACGAATCCCGTGAACCA-TCGAGTCTTTGAACGCAAGTT 323 CGATACTTGG-TGTGAATTGCA-GAATCCCGTGAACCAGTCGAGTCTTTGAACGCAAGTT 321 CGATACTTGG-TGTGAATTGCA-GAATCCCGTGAACCA-TCGAGTCTTTGAACGCAAGTT 353 CGATACTTGG-TGTGAATTGCA-GAATCCCGTGAACCA-TCGAGTCTTTGAACGCAAGTT 336 CGATACTTGG-TGTGAATTGCA-GAATCCCGTGAACCA-TCGAGTCTTTGAACGCAAGTT 353

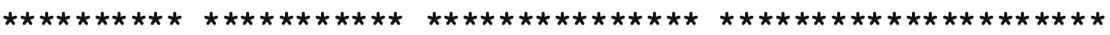

Figura 1. Alinhamento múltiplo parcial entre as sequiências da região ITS $/ 5,8 \mathrm{~S}$ dos acessos de Cratylia argentea com a sequência correspondente de Galactia striata. Os números de cada acesso são mostrados à esquerda de cada sequência, enquanto que as posições dos nucleotídeos são mostradas à direita. As posições contendo deleções de nucleotídeos são mostradas por uma seta, enquanto que as inserções são mostradas em cinza. Os resíduos conservados nas sequências estão indicados com asteriscos $(*)$. 
mostrou que, em todas as sequências ITS de $C$. argentea, a similaridade foi maior na sequência ITS de G. striata, cujo número de acesso no GenBank é AF467049, o que determinou a escolha dessa leguminosa como grupo externo. Após a identificação e edição das regiões ITS para cada um dos 11 acessos de $C$. argentea, com base na similaridade com a sequência homóloga de G. striata, o conteúdo de $\mathrm{G}+\mathrm{C}$ bem como o tamanho das regiões ITS1 e ITS2 foram determinados (Tabela 2). Quanto ao conteúdo de $\mathrm{G}+\mathrm{C}$ das regiões ITS1 e ITS2 dos acessos de $C$. argentea, a Tabela 2 mostra que, em ambas as regiões, ele foi, em média, de 46 a $47 \%$.

Esses resultados estão de acordo com os obtidos na análise desse conteúdo em várias famílias de angiospermas (Moller et al., 1997), em que os conteúdos de $\mathrm{G}+\mathrm{C}$ de ambas as regiões ITS1 e ITS2 foram equivalentes, mas variaram de 47 a $69 \%$ (ITS1) e de 40 a 60\% (ITS2). Na família Fabaceae, representada por uma espécie do gênero Vicia e outra do gênero Vigna, o conteúdo de $\mathrm{G}+\mathrm{C}$ da região ITS1 variou de 52 a $60 \%$, ao passo que, na região ITS2, a variação foi de 50 a $60 \%$ (Baldwin et al., 1995). O fato de o conteúdo de $\mathrm{G}+\mathrm{C}$ das sequências de diversas espécies ser igual, provavelmente, reflete o grau de importância desses espaçadores na maturação das subunidades do ribossomo (Sande, 1992). Quanto ao tamanho das regiões ITS1 e ITS2, existe certa conservação no seu comprimento, em angiospermas. Baldwin (1992) verificou que, na maioria das plantas com flores, as regiões ITS1 e ITS2 são menores que

Tabela 2. Tamanho das regiões ITS1 e ITS2 e percentagem $\mathrm{G}+\mathrm{C}$ dos acessos de Cratylia argentea.

\begin{tabular}{lccccc}
\hline Acesso & \multicolumn{3}{c}{ ITS1 } & & \multicolumn{2}{c}{ ITS2 } \\
\cline { 2 - 3 } \cline { 5 - 6 } & $\begin{array}{c}\text { Tamanho } \\
(\mathrm{pb})\end{array}$ & $\begin{array}{c}\text { Conteúdo de } \\
\mathrm{G}+\mathrm{C}(\%)\end{array}$ & & $\begin{array}{c}\text { Tamanho } \\
(\mathrm{pb})\end{array}$ & $\begin{array}{c}\text { Conteúdo de } \\
\mathrm{G}+\mathrm{C}(\%)\end{array}$ \\
\hline BRA-000591 & 198 & 46,5 & & 206 & 46,6 \\
BRA-000621 & 196 & 46,9 & & 206 & 46,6 \\
BRA-000604 & 198 & 46,5 & & 206 & 46,6 \\
BRA-000639 & 197 & 46,7 & & 206 & 46,6 \\
BRA-000663 & 198 & 46,5 & & 206 & 46,6 \\
BRA-000671 & 198 & 46,5 & & 205 & 46,8 \\
BRA-000680 & 197 & 46,7 & & 205 & 46,8 \\
BRA-000698 & 197 & 46,7 & & 207 & 46,9 \\
BRA-000701 & 198 & 46,5 & & 206 & 46,6 \\
BRA-000728 & 198 & 46,5 & & 206 & 46,6 \\
BRA-000752 & 198 & 46,5 & & 206 & 46,6 \\
Falactia striata $^{(1)}$ & 196 & 52,0 & & 206 & 56,3 \\
\hline
\end{tabular}

${ }^{(1)}$ A sequência completa da região ITS/5,8S de Galactia striata foi obtida do GenBank sob o número de acesso AF467049.
$300 \mathrm{pb}$, com ITS1 variando de 187 a 298 pb e ITS2 de $187 \mathrm{a} 252 \mathrm{pb}$.

Nos acessos de C. argentea estudados, o tamanho do espaçador ITS1 variou de 196 a 198 pb e o tamanho do espaçador ITS2 variou de 205 a 207 pb. Dessa forma, o espaçador ITS1 de todos os acessos de $C$. argentea é menor do que o espaçador ITS2. Em contrapartida, nas sequências ITS disponíveis de espécies das famílias Adoxaceae, Asteraceae, Brassicaceae, Canellaceae, Malvaceae, Onagraceae, Polemoniaceae, Ranunculaceae, Salicaceae, Saxifragaceae, Styracaceae e Winteraceae, o espaçador ITS1 é bem maior que o ITS2. Nas famílias Betulaceae, Cucurbitaceae, Scrophulariaceae e Viscaceae, o ITS2 é maior que o ITS1 (Baldwin, 1992; Baldwin et al., 1995). O alinhamento múltiplo das sequências ITS $/ 5,8 \mathrm{~S}$ dos acessos de $C$. argentea com a sequência correspondente de $G$. striata revelou a presença de deleções e inserções, porém nenhuma mutação do tipo substituição de base - transição ou transversão - foi observada (Figura 1).

Os acessos de $C$. argentea coletados em diferentes municípios dos Estados de Mato Grosso e Goiás foram agrupados em um único clado politômico, separado do grupo externo com um valor de "boostrap" igual a 100 (Figura 2). Esses resultados podem ser explicados pela tese da pouca antiguidade do gênero Cratylia, sugerida pela incipiente divergência morfológica entre suas espécies (Pizarro \& Coradín, 1995). Como os acessos de C. argentea foram coletados em diferentes municípios dos Estados de Mato Grosso e Goiás, onde o ambiente específico da coleta muitas vezes variou (Tabela 1), não foi possível identificar qualquer relação entre os parâmetros ambientais e o padrão de divergência entre as sequências.

Com isso, existe uma grande probabilidade de que as populações desses acessos de $C$. argentea não sejam raças locais, nem ecótipos climáticos ou edáficos. Segundo Grant (1981), raças são sistemas populacionais de espécies que diferem, estatisticamente, de outras populações na sua composição nucleotídica e nas suas características fenotípicas. Os acessos de C. argentea avaliados não diferiram na sua composição nucleotídica da região ITS. Já ecótipos são tipos ou variedades de espécies, localizadas em um ambiente específico, que apresentam variedade molecular ou morfológica (Grant, 1981).

Os fatores climáticos e edáficos podem contribuir para essa variação. O clima da maioria dos municípios 
nos quais os acessos de $C$. argentea foram coletados é semelhante (Tabela 1), mesmo levando-se em consideração a possibilidade de variação dos fatores climáticos ao longo de transectos macrogeográficos. Dessa forma a variação climática não seria, aparentemente, suficiente para produzir diferenciação racial bem definida (Grant, 1981). Não se pode fazer qualquer alusão aos acessos de $C$. argentea como ecótipos edáficos, pois os acessos foram coletados em condições edáficas bastante similares (Tabela 1).

O grau de polimorfismo encontrado em populações pode variar de acordo com a técnica empregada. Anderson et al. (2007), utilizando marcadores moleculares RAPD, encontraram variabilidade genética entre 47 acessos de $C$. argentea. No entanto, a diversidade encontrada foi baixa e homogeneamente distribuída, o que indica extensivo cruzamento. Nos 11 acessos de C. argentea analisados, nenhum polimorfismo do tipo substituição de bases foi observado na região ITS $/ 5,8 \mathrm{~S}$, apesar de essa ser uma região com alta taxa de substituição nucleotídica e indicada para estudos intraespecíficos.

Em relação à análise da região do rDNA $18 \mathrm{~S}$, as sequências das espécies Albizia julibrissin e Bauhinia variegata foram as mais divergentes de todas as outras espécies estudadas, e podem ser tomadas como grupo externo (Figura 3). Dois clados são conspícuos e separados com sustentação de 100\% de bootstrap. Com exceção de três bifurcações de ramos terminais, os

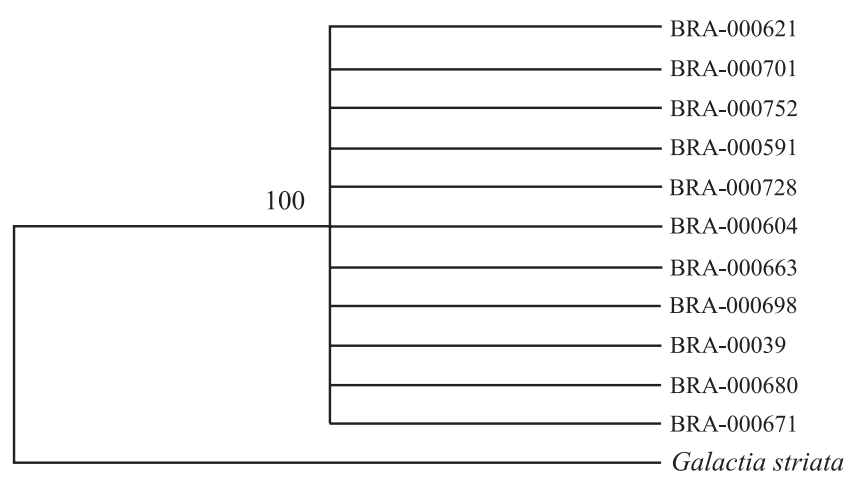

Figura 2. Análise de agrupamento ("neighbor-joining") relacionando as sequências da região ITS/5,8S dos acessos de Cratylia argentea com a sequência homóloga de Galactia striata, construída a partir da matriz de distância (Kimura 2-parâmetros), obtida pelo alinhamento múltiplo entre as sequências. O valor 100, de "bootstrap" (500 replicatas), é mostrado acima do nó que separa o grupo externo das demais sequências. outros valores de "bootstrap" ficaram abaixo de 50\%, o que evidencia que a sequência rDNA $18 \mathrm{~S}$ pode não proporcionar resolução suficiente para a reconstrução filogenética dentro de grupos muito relacionados, como as espécies de Phaseoleae. Seria esperado que Trifolium repens, Astragalus propinquus, Medicago truncatula e Pisum sativum, por não serem Phaseoleae, ocupassem posição mais basal na árvore. No entanto, ficaram agrupadas dentro de um clado com espécies de Phaseoleae.

É pouco provável que essa ambiguidade tenha origem em inconsistências da taxonomia tradicional e esteja mais relacionada à pouca sensibilidade da sequência $18 \mathrm{~S}$, para esse objetivo. Portanto, a reconstrução filogenética desse grupo, com a utilização de sequências nucleotídicas menos conservadas do que o $18 \mathrm{~S}$, poderá gerar árvores filogenéticas com parâmetros de confiabilidade mais robustos.

Cratylia argentea é uma espécie pertencente à subtribo Diocleinae, um táxon de controversas classificações filogenéticas e de incerto reconhecimento natural (Pohill \& Raven, 1981). Embora Lackey (1981) tenha reconhecido 13 gêneros pertencentes à subtribo Diocleinae (Dioclea, Cymbosema, Cleobulia, Canavalia, Pachyrhizus, Macropsychanthus, Luzonia, Camptosema, Cratylia, Collaea, Galactia, Calopogonium, Herpyza), esse autor já havia sugerido que os gêneros Calopogonium, Herpyza e Pachyrhizus tinham características diferentes dos demais gêneros.

$\mathrm{Na}$ determinação do grau de parentesco dessas espécies com base na região do rDNA $18 \mathrm{~S}$, C. argentea ficou agrupada com outras leguminosas que são, indiscutivelmente, membros de Diocleinae (Lackey, 1981), como Cymbosema roseum, Cleobulia multiflora e Dioclea guianensis (Figura 3). Segundo Polhill \& Raven (1981), essas espécies são, portanto, Diocleinae verdadeiras, assim chamadas por apresentarem um reconhecimento natural fácil, fundamentado em caracteres morfológicos. As espécies Calopogonium caeruleum e P. erosus, apesar de serem classificadas como pertencentes a Diocleinae, apresentam-se na árvore filogenética fora do clado que agrupa as Diocleinae verdadeiras (Figura 3). Polhill \& Raven (1981), mesmo ao manter essas duas espécies dentro da subtribo Diocleinae, o fizeram com considerável hesitação, reconhecendo que essas poderiam estar melhor agrupadas de outra maneira.

Esses dados estão de acordo com dendrogramas obtidos a partir de espaçadores do gene rps 16 do 
DNA do cloroplasto (DNAcp) de diversas Phaseoleae (Lee \& Hymowitz, 2001) e de uma inversão repetida, conservada do DNAcp (Doyle \& Doyle, 1993), no qual o complexo Calopogonium-Pachyrhizus está fora de Diocleinae e dentro da subtribo Glycininae. Com base nessas descobertas, Polhill (1994) transferiu Calopogonium e Pachyrhizus da subtribo Diocleinae para Glycininae. Esses resultados também são defendidos por Varela et al. (2004), que analisaram a sequência completa da região ITS (ITS1/5,8S/ITS2) de espécies pertencentes a seis gêneros da subtribo Diocleinae e de algumas espécies dos gêneros Calopognium e Pachyrhizus, e chegaram à conclusão de que esses dois últimos gêneros realmente estão fora do clado que agrupa as Diocleinae verdadeiras. Dessa forma, a análise das sequências do rDNA $18 \mathrm{~S}$ de $C$. argentea com outras leguminosas pode auxiliar no entendimento das relações de parentesco entre espécies de Diocleinae e outras subtribos, o que reafirma resultados alcançados em estudos anteriores, bem como permite estabelecer novos resultados.

$\mathrm{Na}$ análise de agrupamento que usou a região ITS entre os acessos de Cratylia, apesar da ausência de

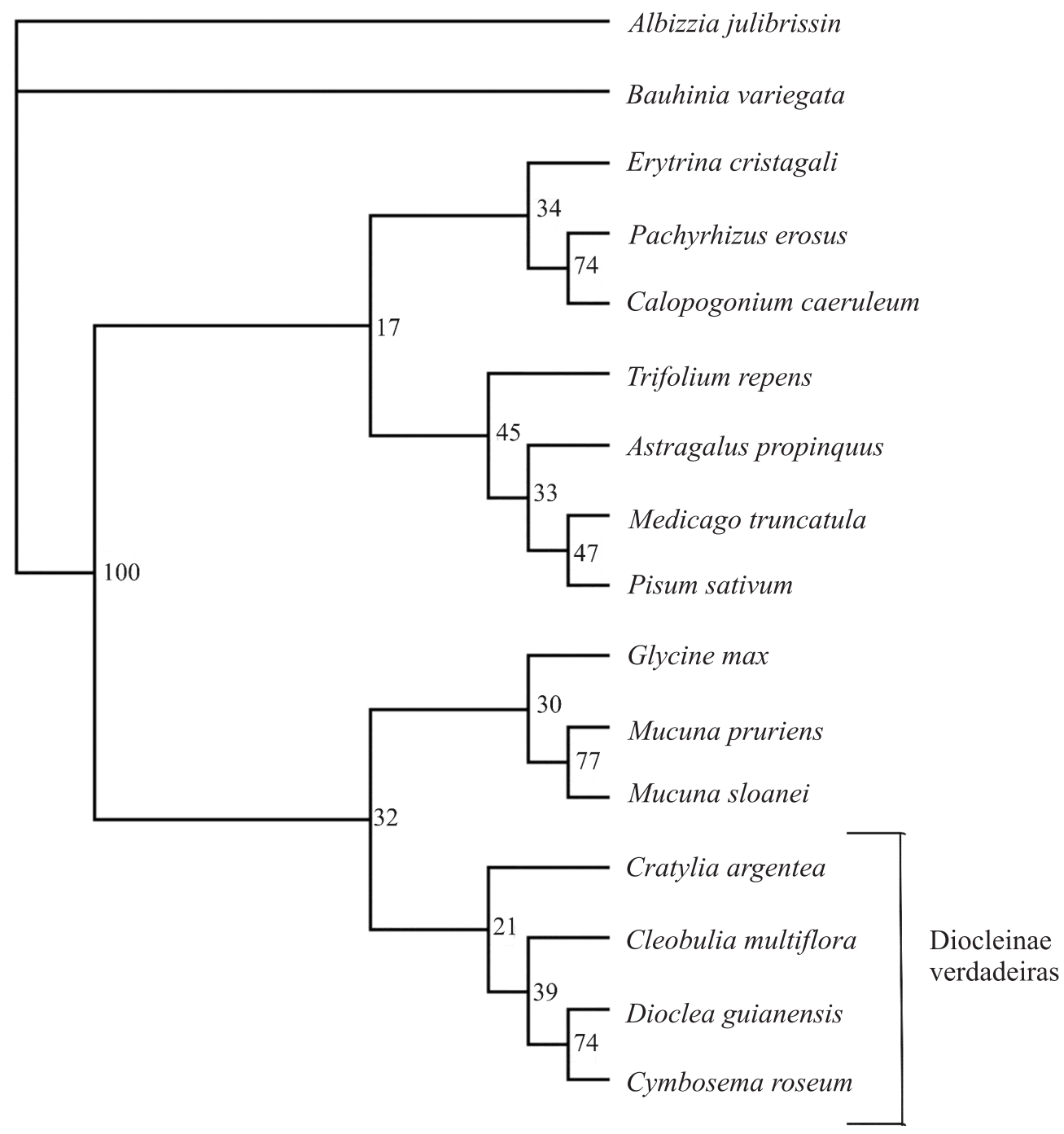

Figura 3. Árvore filogenética ("neighbor-joining") das sequências do rDNA 18S de Cratylia argentea e outras leguminosas, construída a partir de uma matriz de distância (Kimura 2-parâmetros), obtidas através do alinhamento múltiplo das sequências. Albizia julibrissin foi usada como grupo externo. Os valores de "bootstrap" (500 replicatas) são mostrados em cada nó. 
substituição de bases entre as sequências, foram observadas diferenças nessas sequências em relação a inserções e deleções. No entanto, o marcador ITS utilizado neste trabalho não discriminou variabilidade genética entre os acessos. Informações complementares poderiam ser obtidas com o uso de marcadores moleculares do tipo RAPD, RFLP, SNP's.

\section{Conclusões}

1. Acessos de Cratylia argentea podem ser identificados pela comparação das sequências de DNA da região ITS do rDNA nuclear.

2. Não há variabilidade genética entre os acessos de C. argentea analisados.

3. Cratylia argentea é uma espécie pertencente ao grupo das Diocleinae verdadeiras.

\section{Agradecimentos}

À Embrapa Recursos Genéticos e Biotecnologia, pela doação do germoplasma e dados de passaporte dos acessos de C. argentea; ao Dr. José Francisco Montenegro Valls, pelo envio do germoplasma; à Fundação Cearense de Apoio ao Desenvolvimento Científico e Tecnológico, pela concessão de bolsa.

\section{Referências}

ALTSCHUL, S.F.; GISH, W.; MILLER, W.; MYERS, E.W.; LIPMAN, D.J. Basic local alignment search tool. Journal of Molecular Biology, v.215, p.403-410, 1990.

ANDERSON, M.S.; SCHULTZE-KRAFT, R.; PETERS, M.; DUQUE, M.C.; GALEGO, G. Extent and structure of genetic diversity in a collection of the tropical multipurpose shrub legume Cratylia argentea (Desv.) O. Kuntze as revealed by RAPD markers. Eletronic Journal of Biotechnology, v.10, p.386-399, 2007.

ARGEL, P.J.; LASCANO, C.E. Cratylia argêntea (Desvaux) O. Kuntze: una nueva leguminosa arbustiva para suelos ácidos en zonas subhúmedas tropicales. Pasturas Tropicales, v.20, p.37-43, 1998.

BALDWIN, B.G. Phylogenetic utility of the internal transcribed spacers of nuclear ribosomal DNA in plants: an example from the Compositae. Molecular Phylogenetics and Evolution, v.1, p.3-16, 1992.

BALDWIN, B.G.; SANDERSON, M.J.; PORTER, J.M.; WOJCIECHOWSKI, M.F.; CAMPBELL, C.S.; DONOGHUE, M.J. The Its region of nuclear ribosomal DNA: a valuable source of evidence on angiosperm phylogeny. Annals of Missouri Botanical Garden, v.82, p.247-277, 1995.
BECERRA, J.X.; VENABLE, D.L. Nuclear ribosomal, DNA phylogeny and its implications for evolutionary trends in Mexican Burserea (Burseraceae). American Journal of Botany, v.86, p.1047-1057, 1999.

DOYLE, J.J.; DOYLE, J.L. Chloroplast DNA phylogeny of the papilionoid legume tribe phaseoleae. Systematic Botany, v.18, p.309-327, 1993.

FELSENSTEIN, J. PHILIP: phylogenetic inference package. Version 3.5. Seattle: University of Washington, 1993.

FOSTER, G.D.; TWELL, D. Plant gene isolation: principles and practice. New York: Wiley, 1996. 426p.

GRANT, V. Plant speciation. $2^{\text {nd }}$ ed. New York: Columbia University, 1981. 563p.

HALL, T.A. BioEdit: a user-friendly biological sequence alignment editor and analysis program for Windows 95/98/NT. Nucleic Acids Symposium Series, v.41, p.95-98, 1999.

HAO, D.C.; HUANG, B.; YANG, L. Phylogenetic relationships of the genus Taxus inferred from chloroplast intergenic spacer and nuclear coding DNA. Biological \& Pharmaceutical Bulletin, v.31, 260-265, 2008.

KIMURA, M. A simple method for estimating evolutionary rate of base substitutions through comparative studies of nucleotide sequences. Journal of Molecular Evolution, v.16, p.111-120, 1980.

LACKEY, J.A. Tribe 19. Phaseolaceae DC. In: POLHILL, R.M.; RAVEN, P.H. (Ed.). Advances in legume systematics. Kew: Royal Botanic Gardens, 1981. v.1, p.301-327.

LEE, J.; HYMOWITZ, T. A molecular phylogenetic study of the subtribe Glycininae (Leguminosae) derived from the clroroplast DNA rps 16 intron sequences. American Journal of Botany, v.11, p.2064-2073, 2001.

MASS, B.L.Evaluacíon agronómica de Cratylia argentea (Desvaux) O. Kuntze em Colombia. In: In: TALLER DE TRABAJO SOBRE CRATYLIA, 1995, Brasilia. Potencial del genero Cratylia como leguminosa forrajera: memorias. Cali: CIAT, 1995. p.62-74.

MAYER, M.S.; SOLTIS, P.S. Intraspecific phylogeny analysis using ITS sequences: insights from studies of the Streptanthus glandulosus complex (Cruciferae). Systematic Botanical, v.24, p.47-61, 1999.

PAGE, R.D.M. TreeView: an application to display phylogenetic trees on personal computers. Computer Applications in the Biosciences, v.12, p.357-358, 1996.

PIZARRO, E.A.; CORADÍN, L. Potencial del Género Cratylia como leguminosa forrageira. In: TALLER DE TRABAJO SOBRE CRATYLIA, 1995, Brasilia. Potencial del genero Cratylia como leguminosa forrajera: memorias. Cali: CIAT, 1995. p.40-49.

POLHILL, R.M. Classification of the Leguminosae. In: BISBY, F.A.; BUCKINGHAM, J.; HARBONE, J.B. (Ed.). Phytochemical dictionary of the leguminosae. New York: Chapman \& Hall, 1994. p.45-47.

POLHILL, R.M.; RAVEN, P.H. Advances in legume sistematics. Kew: Royal Botanic Gardens, 1981. v.1. 
POSADA, D.; CRANDALL, K.A. MODELTEST: testing the model of DNA substitution. Bioinformatics, v.14, p.817-818, 1998.

QUEIROZ, L.P. de. O gênero Cratylia Mart. Ex Benth. (leguminosae: Papilionoideae: Phaseolae): revisão taxonômica e aspectos biológicos. 1991. 75p. Tese (Mestrado) - Universidade Estadual de Campinas, São Paulo.

QUEIROZ, L.P. de; CORADÍN, L. Biogeografia de Cratylia e áreas prioritárias para coleta. In: TALLER DE TRABAJO SOBRE CRATYLIA, 1995, Brasilia. Potencial del genero Cratylia como leguminosa forrajera: memorias. Cali: CIAT, 1995. p.1-28.

SANDE, C.A.F.M. van der; KWA, M.; NUES, R.W. van; HEERIKHUIZAN, A. van; PLANTA, R.J. Functional analysis of internal transcribed spacer 2 of Saccharomyces cerevisiae ribosomal DNA. Journal of Molecular Biology, v.223, p.899-910, 1992.

SCHMICKL, R.; JORGENSEN, M.H.; BRYSTING, A.K.; KOCH, M.A. The evolutionary history of the Arabidopsis lyrata complex: a hybrid in the amphi-Beringian área closes a large distribution gap and builds up a genetic barrier. BMC Evolutionary Biology, v.10, p.2-18, 2010.

THOMPSON, J.D.; HIGGINS D.G.; GIBSON T.J. CLUSTAL W: improving the sensitivity of progressive multiple sequence alignment through sequence weighting, position-specific gap penalties and weight matrix choice. Nucleic Acids Research, v.22, p.4673-4680, 1994.

TOKUOKA, T. Molecular phylogenetic analysis of Violaceae (Malpighiales) based on plastid and nuclear DNA sequences. Journal of Plant Research, v.121, p.253-260, 2008.

VARELA, E.S.; LIMA, J.P.M.S.; GALDINO, A.S.; PINTO, L. da S.; BEZERRA, W.M.; NUNES, E.P.; ALVES, M.A.O.; GRANGEIRO, T.B.G. Relationships in subtribe Diocleinae (Leguminosae; Papilionoideae) inferred from internal transcribed spacer sequences from nuclear ribosomal DNA. Phytochemistry, v.65, p.59-69, 2004.

VARGAS, S.M.; TORRES, G.A.; SOBRINHO, F.S.; PEREIRA, A.V.; DAVIDE, L.C. Karyotypic studies of Cratylia argentea (Desv.) O. Kuntze and C. mollis mart. ex Benth. (Fabaceae-Papilionoideae). Genetics and Molecular Research, v.6, 707-712, 2007.

XIA, X.; XIE, Z. DAMBE: software package for data analysis in molecular biology and evolution. Journal of Heredity, v.92, p.371-373, 2001 .

YOKOTA, Y.; KAWATA, Y.; IDA, Y.; KATO, A.; TANIFULI, S. Nucleotide sequences of the 5,8S rRNA gene and internal transcribed spacer regions in carrot and broad bean ribossomal DNA. Journal of Molecular Evolution, v.29, p.294-301, 1989.

Recebido em 4 de fevereiro de 2010 e aprovado em 23 de julho de 2010 